\title{
Co-Designing an e-Health Tutorial for Older Adults
}

\author{
Bo Xie $^{1} \quad$ Tom Yeh $^{2} \quad$ Greg Walsh $^{1} \quad$ Ivan Watkins $^{1} \quad$ Man Huang $^{1}$ \\ ${ }^{1}$ College of information Studies, University of Maryland $\quad{ }^{2}$ Dept. of Computer Science, University of Maryland \\ Room 4105 Hornbake \\ College Park, MD 20742 \\ 301-405-2038 \\ A.V. Williams \\ College Park, MD 20742 \\ 301-405-9159 \\ \{boxie, tomyeh, gwalsh, iwatkins, manhuang\}@umd.edu
}

\begin{abstract}
Older adults' ability to access and use electronic health information is generally low, requiring innovative approaches for improvement. An integrated e-tutorial overlays instructions onto Websites. The literature suggests integrated e-tutorials are more effective than paper or video-based tutorials for younger people, but little is known about their effectiveness for older adults. This study explores the applicability of an integrated e-health tutorial for older adults. An integrated e-tutorial, the Online Tutorial Overlay Presenter (OnTOP), added an instructional overlay to the NIHSeniorHealth.gov Website. Overlay features were examined in seven participatory design sessions with seven older adults. Participatory design techniques were used to elicit participants' preferences for tutorial features. Three themes emerged: 1) using contextual cues; 2) tailoring to the learner's literacy level; and 3) enhancing interfaces with multimedia cues. These findings improved the design features of OnTOP. They also generated empirical evidence about the effects of multimedia learning among older adults.
\end{abstract}

\section{Categories and Subject Descriptors}

J.3 [Life and Medical Sciences]: health, Medline.

\section{General Terms}

Design, Human Factors.

\section{Keywords}

Integrated e-tutorial, e-health literacy, participatory design, health information seeking.

\section{INTRODUCTION}

Older adults have the lowest health literacy levels of all adult age groups in the United States [18]. Being able to find useful and reliable health information is an important skill for older adults, whose demand for health information typically increases as they age [32]. Recently, the Internet has become an important source of health information, with $80 \%$ of Internet users seeking health information on the Internet [9]. This presents both new opportunities and challenges for older adults, who typically have

Permission to make digital or hard copies of all or part of this work for personal or classroom use is granted without fee provided that copies are not made or distributed for profit or commercial advantage and that copies bear this notice and the full citation on the first page. To copy otherwise, or republish, to post on servers or to redistribute to lists, requires prior specific permission and/or a fee. low Internet literacy [31,39].

To help older adults take full advantage of the technology, it is essential to develop instructional tools that increase older adults' e-health literacy, or the abilities to access and use electronic health information [26]. Yet, relatively little is known about designing effective tutorials to help older adults develop online health information seeking abilities. This study addresses this gap by incorporating older adults' expertise early in the process, building on the multimedia learning, instructional design, and participatory design literatures.

\section{LITERATURE REVIEW}

\subsection{Multimedia Learning among Older Adults}

A multimedia tutorial contains not only text but also "information in the form of graphics, audio, video, or movies" [11]. The Cognitive Theory of Multimedia Learning holds that multimedia presentations that consist of words (presented as text or audio narration) and pictures (presented as static or animated graphics) foster deeper learning than single-medium presentations [22].

The modality principle [20] asserts that where verbal and pictorial information are necessary for understanding, verbal information should be presented in narrative, rather than written form, so that learners can use auditory cognitive resources to help process verbal information. The redundancy principle [29] posits that using multiple sensory modalities where only one is required to convey understanding imposes an undue cognitive burden. Mayer [23] and Sweller [29] suggest that redundancy can occur when identical information is presented in multiple media forms (e.g., presenting identical verbal information in narrative and written form). The signaling principle [23] suggests that deeper learning occurs when cues are added to a presentation that emphasizes the organization of material, non-redundant information. Mayer [23] argues that signaling reduces demand on cognitive load by focusing the learner's attention on information the learner should attend to. There is preliminary empirical evidence supporting the signaling principle [12,21]. The cognitive aging principle [27] is the only known multimedia learning principle specifically targeting older learners. This principle asserts properly designed multimedia presentations can mitigate age-related cognitive and sensory changes in older adults. It is proposed that two particular approaches to multimedia design may be effective for older adults: learner controlled pacing and part-whole sequencing of information featuring the presentation of pieces of information prior to presenting the complete instruction [27].

With the rapid technological developments in recent years, computer-based multimedia tutorials are increasingly being used for health-related purposes [14,19]. Relatively little is known about what multimedia features of e-health tutorials work for older 
adults, who generally have a great need for health information and services despite low health literacy [18] and computer literacy [4]. These factors make it challenging for older adults to find reliable health information on the Internet [32,31].

\subsection{Tutorial Design Approaches}

Tutorials for older adults typically use print or video demonstrations [7,113]. These media require older adults to alternate between two contexts: the presentation context, which presents information, and the application context, where knowledge is applied during computer operation. Working with two contexts creates challenges. First, older adults must alternate between contexts. After reading an instruction on paper (the presentation context), attention must shift to the computer (the application context) to execute the instruction. Second, older adults must reconcile content between contexts. If an instruction states, "click the OK button", they must locate that button in a different context. Third, older adults rely on short-term memory to transfer instructions between contexts. If the instruction says "type www.nihseniorhealth.gov", they must remember the instruction before they can begin typing. Merging contexts into one application interface provides a technical solution to these challenges (Figure 1).

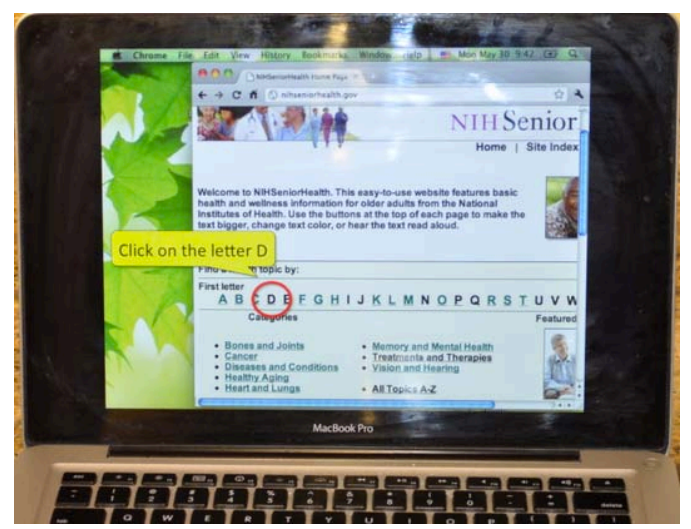

Figure 1. An illustration of the OnTOP contextual tutorial, showing the highlight and the instruction in the same context of the actual web interface.

Instead of presenting the instructions in separate contexts, this integrated solution provides instructions in the target application. For instance, this solution highlights links and provides explanatory text on the screen. Learners remain focused on the screen (rather than a piece of paper) and the application (rather than an application showing a video). The literature indicates this approach benefits user populations like general computer users $[15,2]$ and school children [16]. However, no known study has tested older adult users.

\subsection{Participatory Design}

Older adults are typically "relevant but absent" [1] social groups in the design and development of new technologies. Even though technology plays a critical role in their everyday lives, older adults rarely play a central role in the development process [28]. Technology designers tend to be young adults with a different worldview from older adults, and can have limited understanding of how age-related differences can affect technology use [8]. To ensure the needs and preferences of older adults are fully considered, older adults must be involved in the design and development of technology [38].
Recently, researchers began integrating older adults' input in the design and development of some technologies including: email applications [6], Web browsers [5,17], and informational Web sites [25]. However, no known research has explored the area of ehealth tutorial for older adults.

In the present study, we involved older adults early in the design process, focusing specifically on developing an e-health literacy tutorial for older adults. The present study is part of a larger research project, Electronic Health Information for Lifelong Learners ( $e$ HiLL), which aims to improve older adults' e-health literacy through designing and testing design and educational interventions [34-37]. One key component of the $e$ HiLL project is the cultivation of a core group of older adults interested in helping develop learning materials and strategies to help their age peers learn to use computers to access reliable health information. This group of older adults is called the Older Adult Team (OAT). OAT members formerly participated in an $e$ HiLL intervention [34-37]. These older adults were selected to participate in the OAT because their computer experience exceeded their peers, and because they were willing to commit to the 3 -year duration of the eHiLL-OAT component of the project. The creation of OAT was motivated by our desire to involve older adults as design partners. Since August 2009, the researchers have met with OAT members weekly to collaboratively develop e-health tutorials for older adults. The $e$ HiLL project creates a highly collaborative, educational environment with activities based primarily on participants' feedback.

\section{Method}

\subsection{Research Site}

The participatory design sessions took place at an urban library branch. The library provided computers with high speed Internet access, space, and staff support to facilitate the implementation of this study. This study involved seven sessions conducted between November 2010 and March 2011.

\subsection{Design Partners}

Seven OAT members participated in at least one of the sessions contributing to this paper. Their gender, age, and computer use frequency are summarized in Table 1 below.

Table 1. Participant background information

\begin{tabular}{|l|l|l|}
\hline $\begin{array}{l}\text { Code } \\
\text { name }\end{array}$ & Age & Computer Use Frequency \\
\hline Mr. RH & 88 & Every 2-3 days \\
\hline Ms. MC & 62 & Every day \\
\hline Ms. RG & 72 & Every 2-3 days \\
\hline Ms. JML & 80 & Every day \\
\hline Mr. NJ & 61 & Every 2-3 days \\
\hline Ms. JS & 77 & Every day \\
\hline Ms. SG & 61 & Every 2-3 days \\
\hline
\end{tabular}

\subsection{Design Process}

This study consisted of three participatory design phases: introduction, simulation design, and prototyping. We ran a total of seven two-hour sessions over these three phases (Table 2).

Table 2. Activities used in the co-design phases and sessions of the present study.

\begin{tabular}{|l|l|l|}
\hline Phase & $\begin{array}{l}\text { Sessio } \\
\mathbf{n}\end{array}$ & Activity \\
\hline
\end{tabular}




\begin{tabular}{|l|l|l|}
\hline $\begin{array}{l}\text { Phase 1: } \\
\text { Introduction }\end{array}$ & 1 & $\begin{array}{l}\text { OnTOP presented, solicited } \\
\text { design suggestions }\end{array}$ \\
\cline { 2 - 3 } & 2 & Changes to OnTOP presented \\
\hline $\begin{array}{l}\text { Phase 2: } \\
\text { Simulation } \\
\text { Design }\end{array}$ & 3 & $\begin{array}{l}\text { Recorded participants reading } \\
\text { verbal instructions out loud }\end{array}$ \\
\cline { 2 - 3 } & 4 & $\begin{array}{l}\text { Presented OnTop with } \\
\text { participants' narration }\end{array}$ \\
\cline { 2 - 3 } & 5 & $\begin{array}{l}\text { Physical context: Participants } \\
\text { provide instructions to each } \\
\text { other while seated at the } \\
\text { computer }\end{array}$ \\
\hline $\begin{array}{l}\text { Phase 3: } \\
\text { Prototyping }\end{array}$ & 6 & $\begin{array}{l}\text { Lo-fidelity prototyping using } \\
\text { annotated storyboards }\end{array}$ \\
\cline { 2 - 3 } & 7 & $\begin{array}{l}\text { Target identification using } \\
\text { different types of references }\end{array}$ \\
\hline
\end{tabular}

\subsection{Design Phase 1: Introduction}

The introduction phase familiarized participants with participatory design concepts and practices over two sessions. In the first session, we presented an early prototype of our contextual tutorial tool, Online Tutorial Overlay Presenter (OnTOP), and invited participants to critique and contribute design ideas. The tutorial's content is based on paper-based tutorials we previously developed for teaching older adults to find health information on the NIHSeniorHealth.gov website. [25]

To illustrate the differences between the paper-based tutorial and the contextual tutorial, we first distributed the paper-based tutorial, allowing participants to follow it at their own pace. This tutorial focused on finding diabetes information on NIHSeniorHealth.gov. It has instructions for six steps printed on two pages. Next, we presented the prototype of our computerbased tutorial, OnTOP, which is a contextualized version of the paper-based tutorial. OnTOP displays the same text instructions and visual annotations (e.g., highlight) as the paper-based tutorial, but the instructions and annotations are integrated on top of the website's actual interface. For example, step 5 instructs the user to click on Diabetes. With OnTOP, a highlight appears on the actual word "Diabetes" on the website, which users can click directly. Simultaneously, a text box appears nearby instructing users to "click on Diabetes." Users can click this live link, which takes them directly to the Diabetes web page on the NIHSeniorHealth.gov website (see Figure 1 above). Participants viewed the OnTop tutorial projected on to a large screen. This way, all participants could see it clearly. Then, each participant practiced operating the OnTOP tutorial on the demo machine to gain hands-on experience with the prototype (Figure 2).

Hands-on experience is important because from the users' perspective, watching a contextual tutorial from a distance would be no different from watching a video. The value of contextual tutorial only becomes clear when users operate the interface themselves and see the overlay instructions displayed directly on that interface.

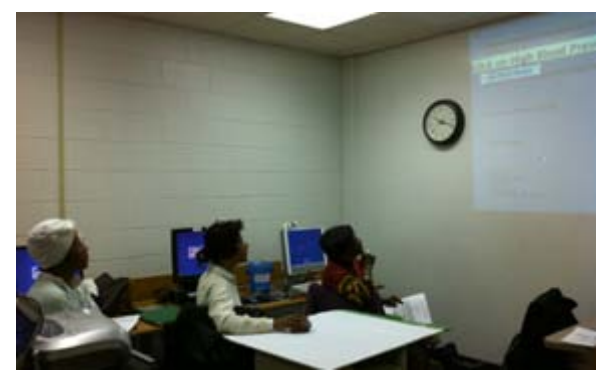

Figure 2. Participants testing an OnTOP prototype.

After using the prototype, we led participants through a co-design activity modeled after comic boarding [24]. Comic boarding is a participatory design technique where children with little participatory design experience describe their ideas to an artist who renders them to paper. We extended this idea by having one researcher sketch design ideas on a large piece of paper as the older adults described what should be on the screen (Figure 3).

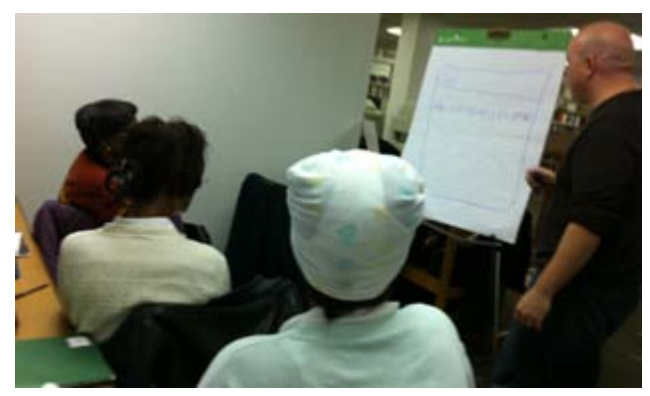

Figure 3. Using the comic-boarding technique to elicit participants' design ideas.

We re-emphasized that the activity's purpose was to gather their feedback and design ideas to help us improve the tutorial they tried. We asked participants to share their likes and dislikes about the prototype. For features they disliked, we solicited suggestions resolving the problem. We concluded this session by debriefing about the co-design process and techniques employed.

After this session, we improved the OnTOP prototype by implementing participants' suggestions that could be implemented given temporal and technical constraints.

In the second session, we aimed to further participants' understanding of participatory design by showing them the changes to the prototype based on their ideas. First, we demonstrated an improved OnTOP prototype that incorporated their design ideas from the previous session. Next, we invited them to offer further critiques and design ideas. This exercise sought to demonstrate to participants their inputs were taken seriously, and that they made important contributions to the design and development of the tutorial.

Similar to the first session, we demonstrated in the improved prototype in the second session. Then,we engaged our participants in a group brainstorming activity to solicit feedback and design ideas about the improved prototype.

\subsection{Design Phase 2: Simulation Design}

Phase 2 involved three sessions. The goal of this phase was to gain insight into the desired features of contextual help through simulation design. We asked participants to speak contextual help aloud to simulate what they may want a computerized tutorial such as OnTOP to present to users. 
In the first session of this phase, participants practiced giving verbal instructions by reading a script out loud (i.e., the paperbased tutorial on which the OnTOP tutorial was built) while we recorded their speech. We chose voice as a delivery method because our participants in the Phase 1 sessions reported voice narration as a desired feature. We used the paper-based tutorial as the script and instructed the participants to read the script, as if they were instructing another person how to find the information. We recorded the speech narrated by each participant using the Audacity voice recording software on a Mac laptop.

In the second session, we asked participants how they would modify the instructions to improve clarity. The participants took turns recording their voices following the improved script. During the break, we integrated the recorded voices into the OnTOP prototype. Participants then listened to and followed the contextual tutorial with voice narration made by themselves and their peers. This activity further demonstrated to participants that their input mattered to the design process. Finally, we solicited feedback from the participants about their experiences. Participants discussed their co-design experience and their likes and dislikes about the prototype. Because of the issues associated with low quality recording in the previous session, we used professional voice recording equipment instead of the laptop's built-in microphone.

In this phase's final session, we asked participants to speak contextual help out loud to their peers without using a script. By now the participants were more familiar with giving and receiving voice-based instructions. We asked participants to work in pairs, with one participant acting as the instructor and the other as the student. The instructor explained to the student how to search for a health topic on NIHSeniorhealth.gov. They then switched roles, repeating the exercise with a different health topic. Finally, we solicited feedback from the participants about their experiences. Participants discussed their participatory design experience and shared their likes and dislikes. We were also interested in participants' retention of the computer knowledge they had learned in these sessions. We asked the participants to explain what strategies they would use to ensure that they remembered the instructions so that they could perform the steps again in the future.

\subsection{Design Phase 3: Prototyping}

In this phase, participants designed paper-based prototypes (i.e., low-fidelity prototypes) using pens, pencils, and highlighters. We conducted two sessions to examine two specific functions of contextual help: providing contextual explanations and referring to target interface elements. In the first session, we examined how a contextual tutorial could integrate contextualized annotations (e.g., a text bubble next to a button) into an interface to aid explanation. We used a low-fidelity prototyping technique to enable the participants to easily express their design ideas. The low-fidelity prototyping technique involved the use of a series of color screenshots of the NIHSeniorhealth.gov website's interface. Each screenshot was printed on a separate piece of paper. We provided participants with pens, pencils, and highlighters and asked them to add their annotations onto the printout interface, as if they were adding these annotations contextually to the real interface on the Web. Participants could also arrange the screenshots in the order they found most effective. The participants were told the tutorial's goal was to provide instructions about locating information about diabetes on the NIHSeniorhealth.gov website for a fictional friend, Mr. Jones, who had never used the NIHSeniorhealth.gov website. This activity's purpose was to help the researchers learn more about how participants would develop and structure a contextual tutorial. We observed closely the types of symbols, language, and highlights participants found useful in designing the tutorials.

In the second session of Phase 3 (i.e., the final session of the present study), we examined how a contextual tutorial could provide different types of references, or cues, to help users identify interface targets on the computer screen. We examined four types of references, including: text only, image only, image with surrounding text, and image with embedded text (Table 3).

Table 3. List of tasks performed by participants during the testing of HealthFinder.gov in Session 7.

\begin{tabular}{|c|c|}
\hline Exemplar reference & $\begin{array}{l}\text { Type of } \\
\text { reference }\end{array}$ \\
\hline $\begin{array}{l}\text { Free interactive tools to check your health, get } \\
\text { personalized advice, and keep track of your progress. }\end{array}$ & Text only \\
\hline & Image only \\
\hline $\begin{array}{l}\text { Diabetes } \\
\text { Do you have diabetes or } \\
\text { pre-diabetes? }\end{array}$ & $\begin{array}{l}\text { Image with } \\
\text { surrounding } \\
\text { text }\end{array}$ \\
\hline & $\begin{array}{l}\text { Image with } \\
\text { embedded } \\
\text { text }\end{array}$ \\
\hline
\end{tabular}

First, we wanted participants to experience these different types of references. We designed target identification tasks using Healthfinder.gov, a website the participants had not previously used. Each identification task consisted of a printout color screenshot and a reference to a target. For example, one task asked participants to "Locate the link for INTERACTIVE TOOLS" on a screenshot of Healthfinder.gov (Figure 4).

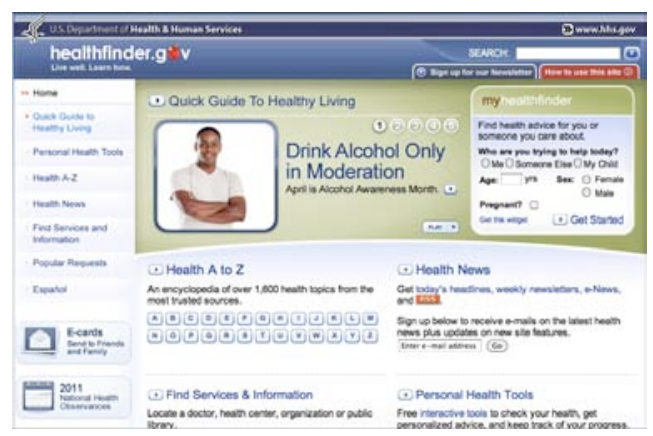

Figure 4. A screenshot of the Healthfinder.gov website.

Because the HealthFinder.gov website was unfamiliar to the participants, they needed to rely on the references to locate objects, rather than relying on their prior knowledge of the website. Using this new website allowed us to assess the effectiveness of these references without the potentially confounding factor of prior knowledge of the website.

Performing the target identification task allowed participants to experience firsthand how helpful each type of reference was. After each task, participants critiqued the effectiveness of the reference type. They were given a hypothetical scenario where they needed 
to improve how interface objects were referenced to ensure others could easily locate the referenced objects. Similar to the previous session, participants contributed their design ideas through lowfidelity prototyping.

\section{DATA ANALYSIS}

Data analysis was guided by the techniques of inductive thematic analysis, that is, "a process of coding the data without trying to fit it into a preexisting coding frame, or the researcher's analytic preconceptions" [3]. Unlike the grounded theory approach that focuses on theory development [10,30], inductive thematic analysis aims to identify salient themes, which was deemed more appropriate given the exploratory nature of the present study and the lack of prior research in the literature.

\section{KEY FINDINGS}

Three major themes were identified from the participatory design sessions:1) contextual cues facilitating learning; 2) the learners' diverse literacy Levels; and 3) enhancing existing interfaces.

\subsection{Contextual Cues Facilitating Learning}

\subsubsection{Gestural cues to help focus attention}

By sharing a physical context participants helped focus each other's attention. This occurred in the simulated contextual help setting, when participants accompanied verbal explanations with gestural cues like pointing at their screen. Mr. RH stated, "we get 'bones and joints' by clicking on the first category," while pointing at the "bones and joint category" on his screen. Mr. RH's use of gestural cues relieved the need for purely verbal explanation and reinforced the verbally conveyed information.

Using gestural cues also allowed participants to use generic terminology in their verbal explanations. For example, Ms. JS stated, "this is a list of disease [s], just like this [link]," while pointing to the link for "hypertension." Ms. JS did not need to provide more specific verbal instruction (e.g., stating, "this is the hypertension link"), because her gesture already conveyed this information. Using gestural cues could be especially useful when participants lack the vocabulary for verbal descriptions, or for words with difficult pronunciation.

Participants confirmed the benefit of gestural cues. Ms. JML stated, "[because] both of us look[ed] at the [same computer] screen, it is easier to point out what you do." For Ms. JML, the shared context of looking at the computer screen allowed her to use gestural cues for explanations.

Recognizing the benefit of gestural cues, we solicited participants' ideas for achieving this effect on the computer. Mr. RH suggested "[an] apple could be the symbol" used to focus users' attention on the screen, presumably because of the symbols' familiarity and easily perceived color. Likewise, Ms. RG offered, "it doesn't hurt to get a flash," suggesting that the apple could flash to attract attention.

\subsubsection{Providing immediate feedback using confirmation cues}

Confirmation cues, provided in context, can help participants recognize when they successfully complete a task. For example, a confirmation cue could indicate a participant clicked the correct link. Participants provided useful design ideas for building confirmation cues on the computer. Ms. RG suggested using audio cues, stating, "like [if] you hit the right place, you will hear a bell," along with visual cues, stating, "if you click it right, then there's something popping out saying, 'you are right.",

\subsection{The learner's diverse literacy levels}

\subsubsection{Providing instructions with appropriate level of details}

Participants suggested the level of details in the tutorial should correspond to the users' knowledge and skill level. Mr. NJ stated, "for [the] person that doesn't know how to use the computer, you need to show [him or her] every function." When Ms. JS provided instruction, she assumed that the user did not know how to use a mouse. More advanced users would not require this detail level. Some participants disliked excessive detail. Mr. RH stated, "I think any more instructions would be an insult."

\subsubsection{Avoiding teaching alternatives}

Teaching multiple methods for completing the same task may confuse users. For example, participants felt providing multiple ways to navigate to a health topic could be confusing. This issue arose because two ways exist for accessing the diabetes health topic. Our participants discussed whether to teach both methods to their age peers, and decided to only teach one. They felt that teaching one method was easier to remember, even if it was less efficient. Ms. RG provided an analogy, stating "just like driving, it is not about speed, it is about knowing what to do."

\subsubsection{Lack of sufficient computer knowledge}

Computer tutorials typically use commonly accepted terms when referencing certain interface elements. This becomes problematic for users with limited computer knowledge. For example, some participants struggled with finding both the text-based links and image-based symbols during Session 7 that focused on locating interface components. They did not understand the text-based links such as "back to top" (Figure 5, red rectangular added) or the image-based symbols (Figure 6).

\section{More information}

The U.S. National Heart, Lung, and Blood Institute has more ahout asthma.

$$
\text { Back to Top }
$$

Figure 5. The text-based link "back to top."

\section{SEARCH:}

Figure 6. The image-based symbol next to the search box.

Conventionally, the "Back to Top" symbol means "if you click here, the page will scroll back to the top of the page" while the search box button is commonly understood to activate the search process. Because our participants lack familiarity with these conventions, they did not understand the functions of these texts or symbols. Similarly, users may have difficulty following explanations using unfamiliar terminology. Some participants felt comfortable using more formal terminology in their explanations, referring to the address bar and the cursor. Other participants relied on lay terms. For instance, Ms. RG referred to the cursor as "the little hand." As with text symbols, using unfamiliar terminology may confuse users who have limited experience with the technology. 
Participants recognized the importance of easily recognizable conventions when developing their own version of the tutorial. When deciding how to number different steps in the tutorial, participants argued over using Roman or Arabic numerals. Participants elected to use Arabic numerals, deciding that too many people might be unfamiliar with Roman numerals. Ms. RG stated, "write the [Arabic] number; people don't know how to do Roman numerals anymore."

\subsection{Enhancing Existing Interfaces}

Contextual help can potentially enhance existing interfaces. Participants identified many ways that can help modify and enhance the existing interface of websites or tutorials.

\subsubsection{Adding custom images and animations}

Participants wanted to enhance the text with illustrative images or animations. For example, Ms. SG stated, "we could use a picture of a syringe or an insulin ball next to diabetes link." She felt that this illustration could clarify the link's meaning and help less literate users. Ms. MC identified an example of an image effectively enhancing the text. She stated, "this picture right here, you know that it is pregnant," referring to the picture showing in Figure 7 below.

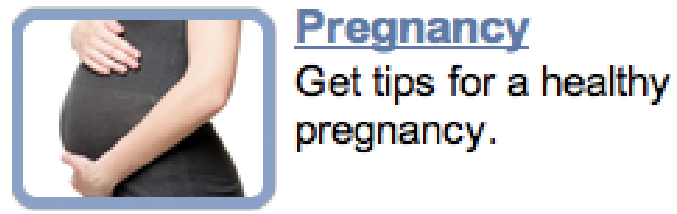

Figure 7. An image from Healthfinder.gov.

Participants also suggested replacing certain text with illustrations. For example, Ms. SG drew a cartoon of two people talking to replace the "speech button". Additionally, she suggested using an illustration of a dizzy person for certain health conditions to indicate illness. Similarly, Mr. RH felt illustrations improved the tutorial's clarity, stating, "we could make a cartoon or something on the side." As with gestural cues, illustrations and images may reduce reliance on purely textual explanations while reinforcing visually conveyed information.

\subsubsection{Adding extra explanations}

Participants suggested adding extra information in some places for clarification. For example, one participant suggested editing the following instruction in the original paper-based tutorial, "Open a Web browser," to "Open a web browser, for example, try Internet Explorer". These participants felt the average user may not know what a "Web browser" was but would know what Internet Explorer was. Providing the additional information could help increase the clarity while reinforcing/delivering the instructional message that Internet Explorer is a type of Web browser. Similarly, the instruction to "click on the link to learn more topics" on the NIHSeniorhealth.gov site was unclear to participants. They did not know this link would navigate to a different website (e.g., MedlinePlus.gov). Adding additional explanatory information could help clarify.

\subsubsection{Adding Reading Aids}

Participants found small or closely spaced text difficult to read. They suggested a text enlargement feature that would aid older users. Mr. RH stated, "I can't enlarge text on all websites, [but if] someone has a magnifying glass ... I would use that all the time." While most modern browsers support this feature, users, particularly those who have limited experience with the technology, may not necessarily know about this built-in feature. A possible solution might be to automatically detect when the font is too small and instruct the users on enlarging the font.

\section{DISCUSSION}

This study contributes to the multimedia learning literature, particularly to four design principles discussed above. Our participants favored auditory presentation of the instructions (i.e., narration using their own voices), lending support for the modality principle [20]. Interestingly, their positive attitude towards the auditory presentation mode persists even when the narration is identical to the written instructions. At the first glance, this may seem contradictory to the redundancy principle that predicts negative impact when identical information is presented in multiple media forms [23,29]. A closer look, however, suggests a new possibility: that is, identical information presented visually and verbally may not necessarily be "redundant" to the learners. In another $e$ HiLL project conducted with older adults in the same community as those participated in this study, the randomized controlled experiments revealed no statistically significant difference in the learning outcomes of older adults in groups receiving visual only instructions and those in groups receiving visual plus auditory instructions [34].

One possible reason may lie in the library setting where the study was conducted [34]. At the library site, due to a lack of separate computer lab facility, all sessions were held in a public area where library staff and other patrons were constantly walking by and sometimes talking. These distractions might have interfered with older adults' learning, particularly for those under the visual only experimental condition. In comparison, for the visual plus auditory condition, because the auditory information was presented directly to each participant via individual headphones, hearing the narration might have been conducive to participants staying focused. In this sense, the information presented via the auditory channel might not have been "redundant" to that presented visually [34]. Because the present study was conducted at the same library site with the same physical arrangements, it might have also been affected by the same environmental factors. It will be interesting in future research to explore if and how environmental factors such as noise might affect, for instance, under what specific circumstances the information presented via what channel(s) is, or is not, redundant.

Our findings lend support to the signaling principle [22] which promotes the use of cues to focus learner attention and enhance learning. Our participants relied on gestural and confirmation cues to deliver the instructions to peers, and recommended ways to incorporate these cues into the computer-based OnTOP tutorial. These findings are in line with prior research that also supported the signaling principle [12,21]. The mechanism behind the potential effectiveness of contextual cues, as Mayer [23] argues, is that signaling reduces cognitive load by helping the learner to focus on the key information necessary for learning.

The multimedia learning literature focuses primarily on younger learners in formal educational settings [22]. Our findings suggest the theories and principles of multimedia learning may be generalized to the older population in informal settings. Our findings also provide empirical evidence for the proposed design approaches under the cognitive aging principle [27]: i.e., the OnTOP tutorial ensures learner controlled pacing, which can accommodate individual differences in cognitive abilities [27]. 


\subsection{Co-design methods}

Comic boarding was originally developed to promote participatory design among children by providing an on-site artist to illustrate ideas as they are mentioned and draw storyboards [24]. Our study, by testing this technique among older adults, provides evidence that the comic boarding technique may be applied to other social groups that also have limited participatory design experience. At the end of this session, the older adults expressed that they would like to use the tools and draw for themselves at a later session. This seemed to be in contrast to earlier findings of co-designing with older adults [38]. This could be because of differences in personalities or that the older adults became more confident in their skills as co-designers and may have felt as though their input would matter.

\subsection{Limitations and future directions}

This study had some limitations. It involved a small, convenience sample that may not be representative of the older population nationwide and should not be generalized without caution. Also, the sessions were conducted in a naturalistic setting familiar to older adults: i.e., a public library in their own communities, which had obvious advantages including increasing participation and making the results more readily transferable than those generated in a research laboratory. However, this choice of the research site also introduced challenges in terms of controlling for potentially confounding environmental variables. It will be interesting in future research to examine if the results might be duplicated in different environments.

Participants lacked the technical ability to implement these enhancements because enhancements can only be implemented through programming. A desired feature of the contextual tutorial tool would be to enable the user to add custom annotations without programming. One possible solution would be to record users' gestures and voices as they speak aloud the enhancements they like to introduce to a website (as our participants did in the current study) and convert the gestures and voices into content that can be presented contextually in that website by OnTOP.

\section{CONCLUSION}

Using multiple participatory design techniques, we explored older adults' preferences for design features of an integrated e-tutorial, OnTOP. The findings suggest that the use of contextual cues may facilitate learning. Due to the diversity of learner literacy levels, it is important to provide tailored tutorials to accommodate different learner's literacy Level. Further, tutorial interfaces may be enhanced with the addition of multimedia cues: e.g., using images of everyday objects to replace unfamiliar abstract computer symbols. These findings helped improve the design features of the OnTOP. Importantly, these findings also contribute to the multimedia learning literature by generating empirical evidence about the effects of multimedia learning among older adults, a previously understudied population in the literature, and by raising interesting questions worthy of further examination (e.g., under what circumstances might information be "redundant" to older adults' learning).

\section{ACKNOWLEDGEMENTS}

We thank the older adults who participated in this study and the Hyattsville librarians who helped with the logistics. The Electronic Health Information for Lifelong Learners ( $e$ HiLL) project is funded by an Institute of Museum and Library Services Faculty Early Career Development Award to Bo Xie.

\section{REFERENCES}

[1] Berg, A.-J. (1999). A gendered socio-technical construction: the smart house. In D. MacKenzie \& J. Wajcman (Eds.), The Social Shaping of Technology (2nd ed., pp. 301-313). Buckingham: Open University Press (pp. 225-239). Leiden: DSWO Press.

[2] Bergman, L., Castelli, V., Lau, T., and Oblinger, D. DocWizards: A System for Authoring Follow-me Documentation Wizards. In Proceedings of the 18th annual ACM symposium on User Interface Software and Technology - UIST 05, ACM Press (2005), 191.

[3] Braun, V., \& Clarke, V. (2006). Using thematic analysis in psychology. Qualitative Research in Psychology, 3, 77-101.

[4] Czaja, S. J., Charness, N., Fisk, A. D., Hertzog, C., Nair, S. N., Rogers, W. A., et al. (2006). Factors predicting the use of technology: Findings from the Center for Research and Education on Aging and Technology Enhancement (CREATE). Psychology and Aging, 21(2), 333-352.

[5] Dickinson, A., Gregor, P., Mcivr, L., Hill, R. L. \& Milne, S. (2005). The Non Browser: helping older novice computer users to access the web. Accessible Design in the Digital World Conference. Dundee, Scotland.

[6] Dickinson, A., Newell, A. F., Smith, M. J. \& Hill, R. L. (2005). Introducing the Internet to the over-60s: Developing an email system for older novice computer users. Interacting with Computers, 17, 621-642.

[7] Echt, K. V., Morrell, R. W., \& Park, D. C. (1998). Effects of age and training formats on basic computer skill acquisition in older adults. Educational Gerontology, 24(1), 3-25.

[8] Eisma, R., Dickinson, A., Goodman, J., Mival, O., Syme, A. \& Tiwari, L. (2003). Mutual inspiration in the development of new technology for older people. INCLUDE. London, UK.

[9] Fox, S. (2011). Health Topics. http://www.pewinternet.org/ Reports/2011/HealthTopics.aspx.

[10] Glaser, B. G., \& Strauss, A. L. (1967). The discovery of grounded theory: Strategies for qualitative research. Chicago: Aldine.

[11] Greenlaw, R. \& Hepp, E. (1999). In-line/On-line: Fundamentals of the Internet and the World Wide Web. Boston: McGraw-Hill.

[12] Harp, S. F., \& Mayer, R. E. (1998). How seductive details do their damage: A theory of cognitive interest in science learning. Journal of Educational Psychology, 90, 414-434.

[13] Hawthorn, D. (2007). Interface design and engagement with older people. Behaviour \& Information Technology, 26(4), 333-341.

[14] Hinchliffe, A. \& Mummery, W.K. (2008). Applying usability testing techniques to improve a health promotion website. Health Promotion Journal of Australia, 19(1), 29-35.

[15] Kang, H. and Plaisant, C. New approaches to help users get started with visual interfaces: multi-layered interfaces and integrated initial guidance. In Proceedings of the 2003 Annual National Conference on Digital Government Research, (2003).

[16] Kelleher, C. and Pausch, R. Stencils-Based Tutorials: Design and Evaluation. In Proceedings of the SIGCHI Conference 
on Human Factors in Computing Systems - CHI 05, ACM Press (2005), 541.

[17] Kurniawan, S. H., King, A., Evans, D. G. \& Blenkhorn, P. (2006). Personalising web page presentation for older people. Interacting with Computers, 18, 457-477.

[18] Kutner, M., Greenberg, E., Jin, Y., \& Paulsen, C. (2006). The Health Literacy of America's Adults: Results From the 2003 National Assessment of Adult Literacy (NCES 2006-483). U.S. Department of Education. Washington DC: National Center for Education Statistics.

[19] Lin, C.A., Neafsey, P.J., \& Strickler, Z. (2009). Usability testing by older adults of a computer-mediated health communication program. Journal of Health Communication, 14(2), 102-118.

[20] Low, R. \& Sweller, J. (2005). The Modality Principle in Multimedia Learning. In R. Mayer (Ed.), The Cambridge Handbook of Multimedia Learning (pp. 183-200). New York: Cambridge University Press.

[21] Mautone, P. D., \& Mayer, R. E. (2001). Signaling as a cognitive guide in multimedia learning. Journal of Educational Psychology, 93, 377-389.

[22] Mayer, R. (2005a). The Cambridge Handbook of Multimedia Learning (ed.). Cambridge University Press: New York.

[23] Mayer, R. (2005b) Principles for Reducing Extraneous Processing in Multimedia Learning: Coherence, Signaling, Redundancy, Spatial Contiguity, and Temporal Contiguity Principles. In R. Mayer (Ed.), The Cambridge Handbook of Multimedia Learning (pp. 183-200). New York: Cambridge University Press.

[24] Moraveji, N., Li, J., Ding, J., O’Kelley, P., \& Woolf, S. (2007). Comicboarding: using comics as proxies for participatory design with children. Proceedings of the SIGCHI conference on Human factors in computing systems, CHI '07 (p. 1371-1374). New York, NY, USA: ACM. doi:10.1145/1240624.1240832

[25] Morrell, R. W. (2005). http://www.nihseniorhealth.gov: the process of construction and revision in the development of a model web site for use by older adults. Universal Access in the Information Society, 4(1), 24 - 38.

[26] Norman, C. D., \& Skinner, H. A. (2006). eHealth literacy: essential skills for consumer health in a networked world. Journal of Medical Internet Research, 8(2), e9.

[27] Paas, F., Van Gerven, P., \& Tabbers, H. (2005). The Cognitive Aging Principle in Multimedia Learning. In R. Mayer (Ed.), The Cambridge Handbook of Multimedia
Learning (pp. 339-351). New York: Cambridge University Press.

[28] Paquette, S. \& Xie, B. (2010). The Relevance of Elderly Technology Users in Healthcare Knowledge Creation and Innovation: A Case Study. In Proceedings of the $43^{\text {rd }}$ Hawaii International Conference on Systems Sciences. January 5-8, 2010, Hawaii.

[29] Sweller, J. (2005). The Redundancy Principle in Multimedia Learning. In R. Mayer (Ed.), The Cambridge Handbook of Multimedia Learning (pp. 159-167). New York: Cambridge University Press.

[30] Strauss, A. L., \& Corbin, J. (1998). Basics of qualitative research: Techniques and procedures for developing grounded theory (2nd ed.). Thousand Oaks, CA: Sage.

[31] Xie, B. (2008). Older adults, health information, and the Internet. ACM Interactions, 15(4), 44-46.

[32] Xie, B. (2009). Older adults' health information wants in the Internet age: Implications for patient-provider relationships. Journal of Health Communication, 14(6), 510-524.

[33] Xie, B. (2011). Older adults, e-health literacy, and collaborative learning: An experimental study. Journal of the American Society for Information Science and Technology (JASIST), 62(5): 933-946.

[34] Xie, B. (2011). Experimenting on the impact of learning methods and information presentation channels on older adults' e-health literacy. Journal of the American Society for Information Science and Technology (JASIST) 62(9), 17971807.

[35] Xie, B., \& Bugg, J. M. (2009). Public library computer training for older adults to access high-quality Internet health information. Library \& Information Science Research, 31, 155-162.

[36] Xie, B. (2011). Effects of an e-health literacy intervention for older adults. Journal of Medical Internet Research, 13(4):e90. Full text: http://www.jmir.org/2011/4/e90/.

[37] Xie, B. (in press). Improving older adults' e-health literacy through computer training using NIH online resources. Library \& Information Science Research.

[38] Xie, B., Druin, A., Fails, J., Massey, S., Golub, E., Franckel, S., \& Schneider, K. (in press). Connecting generations: developing co-design methods for older adults and children. Behaviour \& Information Technology.

[39] Zickhur, K. (2010). Generations 2010. http://www.pewinternet.org/Reports/2010/Generations2010/Introduction/Generations-online-and-offline.asp 